\title{
Splenic rupture following transcatheter arterial embolization of splenic artery pseudoaneurysm caused by acute pancreatitis
}

\author{
Jung Woo Lee, Tae Nyeun Kim, Sung Bum Kim, and Kook Hyun Kim
}

Division of Gastroenterology and Hepatology, Department of Internal Medicine, Yeungnam University College of Medicine, Daegu, Korea

Received: July 23, 2015

Revised : September 24, 2015

Accepted: September 24, 2015

\section{Correspondence to}

Tae Nyeun Kim, M.D.

Tel: +82-53-620-3842

Fax: +82-53-654-8386

E-mail: tnkim@yu.ac.kr
A 32-year-old man visited our emergency department complaining of severe left upper quadrant abdominal pain. He had history of alcoholic acute pancreatitis complicated with multiple peripancreatic pseudocysts and splenic vein thrombosis with splenic artery pseudoaneurysm and obliteration of the pseudoaneurysm was done by transarterial coil embolization 6 months ago. An abdominal computerized tomography scan at admission showed decreased perfusion at lower pole of spleen with focal splenic infarct, fluid collection at adjacent areas of pancreatic tail and stomach, and pseudocyst at pancreatic tail (Fig. 1). The patient received conservative treatment. On the

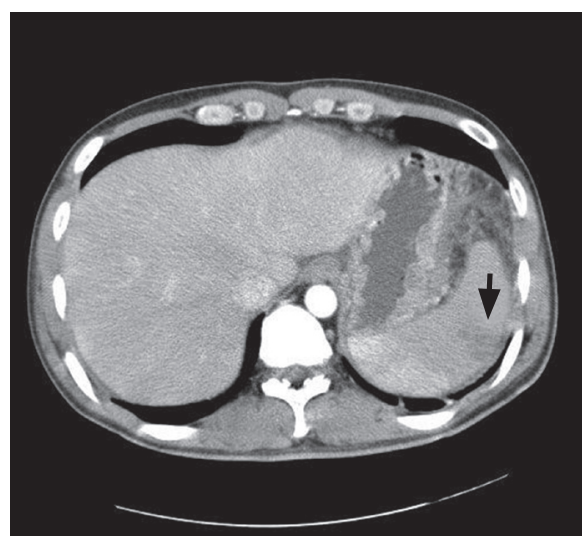

Figure 1. Abdominal computed tomography scan at admission. Decreased splenic perfusion and focal splenic infarct (arrow) were noted. 16th day of hospitalization, sudden onset of severe abdominal pain developed. His blood pressure declined to 80/50 $\mathrm{mmHg}$, and remained to be persistently below $90 \mathrm{mmHg}$ despite intravenous fluid therapy and use of inotropics. An emergent abdominal computed tomography scan revealed hemoperitoneum with active bleeding around splenic hilum and pancreatic tail (Fig. 2). An emergency surgery was performed and pin point bleeding from lateral wall of spleen was noted. Splenectomy with clipping of short gastric and splenic vessels was done. The patient was discharged without complication on the 1oth postoperative day.

Pseudoaneurysm of the splenic ar-

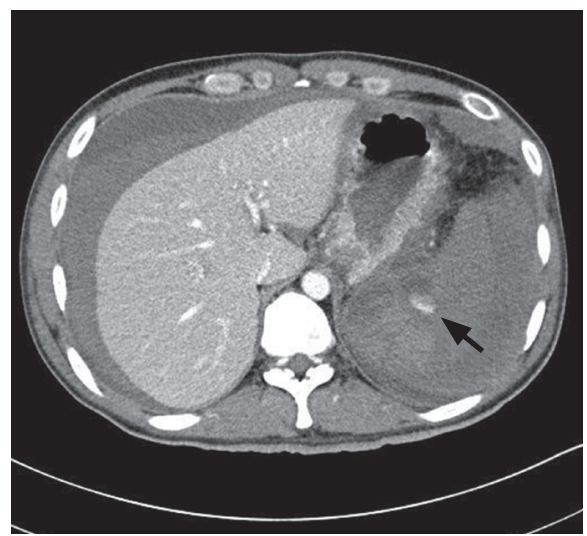

Figure 2. Abdominal computed tomography scan at the time of hemoperitoneum. Active bleeding around the spleen (arrow) and hemoperitoneum had developed. 
tery, infarction, and rupture of the spleen are uncommon complications of acute or chronic pancreatitis. In our case, splenic vein thrombosis and adhesion around the pancreas and spleen due to previous severe acute pancreatitis as well as decreased splenic perfusion due to embolization may have contributed to development of splenic infarction with rupture. Treatment of a ruptured spleen depends primarily on hemodynamic stability of the patient, followed by extent of spleen injury and degree of hemoperitoneum. In our case, the patient underwent surgery due to hemodynamic instability. We report a case of splenic infarction and subsequent splenic rupture, treated by open splenectomy, following transcatheter embolization of an asymptomatic splenic artery pseudoaneurysm associated with acute pancreatitis.

\section{Conflict of interest}

No potential conflict of interest relevant to this article was reported. 